\title{
Hans Heinrich Brunner 1944-2010
}

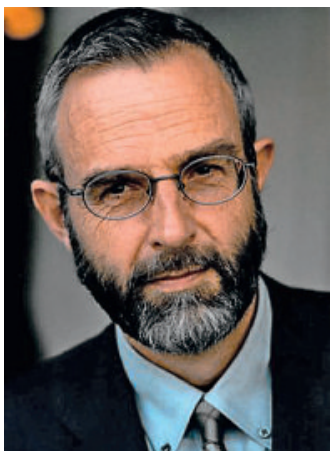

Dr. med.

Hans Heinrich Brunner, $\mathrm{MPH}$, Präsident der FMH von 1994 bis 2004.
Die schweizerische Ärzteschaft hat in diesen Tagen eine ihrer bedeutendsten Persönlichkeiten verloren. Hans Heinrich Brunner ist gestorben. Er war ohne Zweifel eine der Lichtgestalten der Gesundheitspolitik in unserem Land. Kaum jemand hat das schweizerische Gesundheitswesen in den vergangenen zwanzig Jahren so geprägt, so tiefe Spuren hinterlassen wie er - kaum jemand war wohl auch so umstritten und hat neben hellem Licht auch Schatten geworfen.

Gedanken an Leben und Tod Hans Heinrich Brunners spannen die Assoziation an ein antikes Drama. Heroische, in unerbittliche Kämpfe verwickelte Gestalten, übermenschliche Kraftanstrengungen, Mut, Zorn, List und Tücke, das Streben nach dem Absoluten, mythische Überhöhungen und die Konfrontation mit den unverrückbaren Wahrheiten des Menschseins: Von alldem war auch dieses Leben gefüllt. Die Antike war ihm auch nicht fremd. Ja, ganz direkt prägten sprachliche Reminiszenzen seine Veröffentlichungen, etwa durch den Gebrauch lateinischer Aphorismen oder der Verwendung von Gräzismen, wenn er von der «idiosynkratischen Reaktion» auf Prämienerhöhungen sprach, oder Anhänger (seiner Gegner) schlicht als «Heloten» bezeichnete. Auch in der Körperlichkeit war er ein Titan. Ein Fels von einem Mann. Manche sahen in ihm vielleicht eher den bärtigen, unbeugsamen Bergler, ein Abbild Tells im 20. Jahrhundert. Er liebte und kultivierte dieses Selbstbild wetterfester Vierschrötigkeit mit schwarzem Rollkragenpullover, Rucksack und Bergschuhen.

Doch manches war bei HHB anders, als es auf den ersten Blick schien. Wohl war er überzeugter Innerschweizer und diente seiner Gemeinde Vitznau während einiger Jahre in der Schulpflege und seinem Kanton als Kantonsrat - allerdings nicht als katholisch konservativer Roter, wie es der regionalen Mehrheit entsprochen hätte, sondern als reformierter liberaler Schwarzer, wie die Luzerner Tradition die Parteien in Umkehr der Farbsymbolik benannte. Doch letztlich war er auch kein Ur-Innerschweizer, sondern Bürger des Oberwalliser Ortes Binn, aufgewachsen in Olten, dem Knotenpunkt des Schweizerischen Bahnnetzes, und später im benachbarten Zofingen. Der Umstand, dass ein Zweig seiner Vorfahren auf den preussischen General von Clausewitz zurückführte, war für ihn mehr als beiläufige Koketterie: Dem Vordenker militärischer Strategie und taktischer Kriegsführung fühlte er sich auch in der Gegenwart verpflichtet - vielleicht zog auch ein kühler Windzug protestantisch-preussi- schen Arbeitsethos durch seine Kindheit. Die prägende Person der Jugend war gewiss der Vater: Heinrich Brunner, Generaldirektor beim Verlagshaus Ringier und einer der Begründer der ersten Schweizerischen Boulevardzeitung. Von ihm hatte er einerseits unternehmerisches Flair und wohl auch Durchsetzungsvermögen mitbekommen und andererseits direkten Einblick und Verständnis für die Mechanismen des Aktualitätsjournalismus. Beides sollte ihm später zugutekommen.

Schon für den privilegiert heranwachsenden Schüler der Aarauer Kantonsschule war der flache Hedonismus der 60er Jahre kein Lebensziel. Er begeisterte sich für Mathematik, besuchte freiwillige Kurse in Hebräisch und Philosophie, schlug sich die Nächte mit Diskussionen um das Wesen der letzen Dinge um die Ohren, suchte schon damals das Absolute. Zeitlebens blieben Geschichte, Philosophie und Soziologie; waren geistige Herausforderung und intellektuelle Prägnanz seine Welt. Zur Entspannung las er Luhmann oder Wittgenstein, setzte sich mit Habermas, Bloch oder Dahrendorf auseinander. Er kannte die europäische Ideen- und die schweizerische Nationalgeschichte. Er war ein hochkultivierter Mann - allerdings wohl mehr des Geistes, und weniger des Umgangs.

Brunner absolvierte das Studium der Medizin an den Universitäten Basel und Zürich und die anschliessende Weiterbildung in Innerer Medizin und Kardiologie am Universitätsspital Zürich. In jene Zeit fielen neue Entwicklungen im Bereich der Kardiologie, die Echokardiographie kündigte sich an, doch ein akademischer Weg war seine Sache nicht. Zu unruhig, wohl auch zu unabhängig, war sein Geist, als dass er sich den Gepflogenheiten einer universitären Hierarchie untergeordnet hätte. Im Jahr 1980 eröffnete Hans Heinrich Brunner eine Praxis im Zentrum von Luzern, als Internist mit der Subspezialität (das gab es damals noch) Kardiologie. Hier lernte er die Situation der niedergelassenen Ärzteschaft kennen, erfuhr aus eigener Ansicht die Nöte und beruflichen Mechanismen des freien Arztes. Er war zeitlebens froh um diese Erfahrung und kehrte in den letzten Jahren auch nochmals in diese Rolle zurück. Doch die Vorstellung, nun bis zur Pensionierung seinen Einflussbereich auf die eigenen Praxisräumlichkeiten zu beschränken, behagte ihm nicht. Aussergewöhnlich und bemerkenswert genug, gelang ihm im Jahr 1988 aus der Praxis der Sprung auf die Stelle des Co-Chefarztes für Innere Medizin des Spitals Sursee. Hier fand er das Umfeld seiner ärztlichen Entfaltung zum allgemeininternistischen Generalis- 
ten, der nicht nur die kardiologischen Untersuchungen und Abklärungen vornahm, sondern auch den Ultraschall und die Endoskopie pflegte, Patienten betreute und Assistenzärzte an die Medizin heranführte.

Ab 1984 öffnete sich mit der Wahl in den Zentralvorstand der FMH ein neues Feld. In der Folge wurden Fragen des ärztlichen Tarifwesens zu seinem Spezialgebiet, ja letztlich zu seinem Lebenswerk. In den 80er Jahren gelang es ihm, die Ärzteschaft von der Notwendigkeit zu überzeugen, das historisch auf der Grundlage von «Basar-Verhandlungen» entstandene Tarifwesen einer Gesamtrevision zu unterziehen. GRAT war das Akronym, das diesem Projekt den Namen gab und in seinem Innersten von der Idee getragen war, das gesamte ärztliche Tarifsystem auf eine rationale, betriebswirtschaftliche Basis zu stellen. Das war schon zu Beginn eine Aufgabe gigantischen Ausmasses, ursprünglich nur für den UV-, IV-, MV-Arzttarif gedacht, die sich im Verlauf der Zeit durch den Einbezug der Krankenversicherer in das Projekt TARMED noch erweiterte. Brunner war von Anfang bis zum Ende der spiritus rector, derjenige, der es zu Beginn mit den Partnern auf Seiten der Versicherer erdacht hatte, der mit Delegierten der Fachgesellschaften die Einzelleistungen erarbeitete und Inkongruenzen und Widersprüche unter den über 6000 Einzelleistungspositionen ausglich, verhandelte und in der Endphase auch unerbittlich erstritt. Wohl kaum jemand kannte die Geschichte, vor allem aber auch die inneren Zusammenhänge des TARMED so gut wie er. Die Arbeit an diesem epochalen Tarifwerk wurde zu seiner zentralen Lebensleistung. Er wusste sehr wohl um die individuelle und die gesamtwirtschaftliche Verantwortung, die in der Einkommens-bestimmenden Modellierung eines Vergütungssystems in seinen Händen lag - und er mag diese Macht auch gelegentlich genossen haben. Doch war es nicht das, was ihn in erster Linie umtrieb. Seine innere Faszination galt der Vorstellung, ein in sich kongruentes Regelwerk zu schaffen, einen Mechanismus, wie ein mechanisches Uhrwerk, der Ordnung und Ausgleich schafft. Er sah sich gern in der Rolle des Inge-

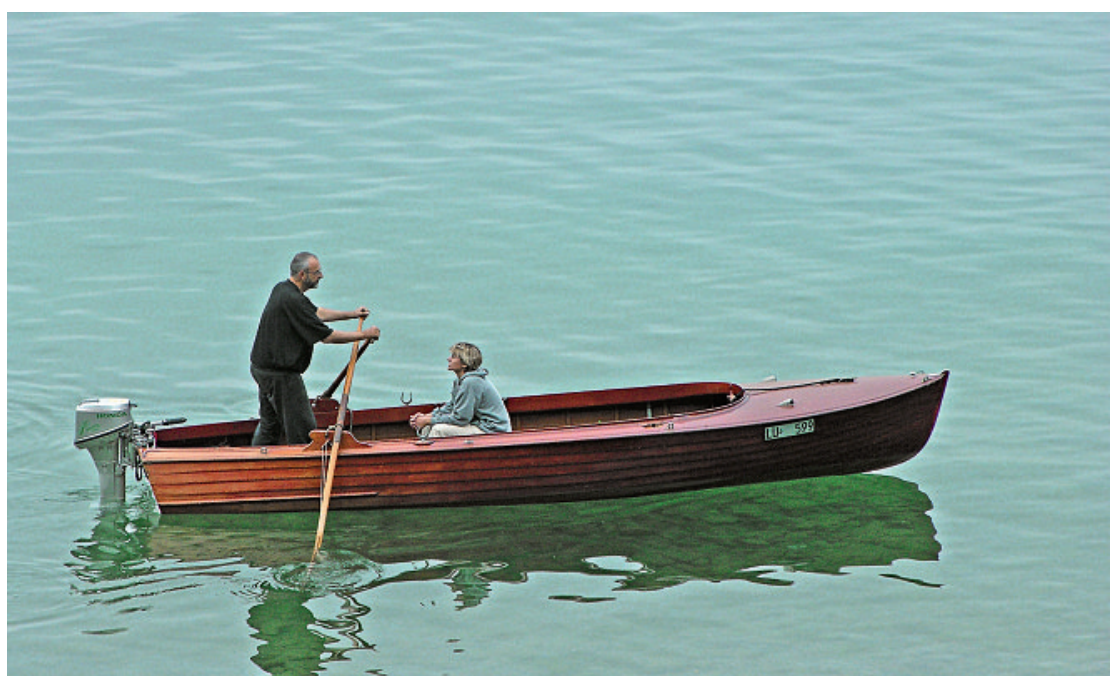

nieurs und Mechanikers, der die Schrauben anzog und die Ventile einstellte, zumindest im Groben, denn wenn er die grundsätzlichen Zusammenhänge erfasst hatte, wandte er sich, rastlos wie er war, gern einem neuen Thema zu und überliess die Feinarbeit anderen.

*

Entwicklung und Einführung des TARMED waren das Leitmotiv seiner Zeit als FMH-Präsident von 1994 bis 2004, aber nicht das einzige, das blieb. Als Ausgleich der die FMH erfassenden zentrifugalen Kräfte erlebte der Verband 1998 eine grundlegende Strukturreform, die Arbeitsweise einer in gewissem Sinne noch im Ancien Régime verhafteten Standesvertretung wurde auf die Bedürfnisse einer modernen Berufsorganisation umgekrempelt, am sichtbarsten im Einzug der modernen Informationstechnologien. Nicht unumstritten, aber in vieler Hinsicht wegweisend und noch heute von Bedeutung, war das Eingehen strategischer Partnerschaften aus denen sich zum Teil Organisationen und Unternehmen entwickelt haben, die den Interessen der Ärzteschaft flankierend zur Seite stehen. HIN, BlueCare, EMH, NewIndex, College M: Alle entstanden im Umfeld seiner strategischen Ziele, zu denen als allererstes gehörte, die Unabhängigkeit und Handlungskompetenz der Ärzteschaft im politischen Raum zu erhalten und zu stärken. Wie gut ihm dies gelungen ist, werden Einzelne unterschiedlich beurteilen; wer ihn miterlebt hat, weiss, dass er, wo immer sie sich boten, Handlungsspielräume genutzt, sie bis an die Grenzen - und auch manchmal darüber hinaus - ausgelotet hat.

Er war nicht der geborene Lobbyist, aber im Kampf für die Sache konnte Hans Heinrich Brunner über sich hinauswachsen. So wenig er der gewinnende Causeur auf gesellschaftlichem Parkett war, so wirkungsvoll konnte er in der direkten sachlichen Auseinandersetzung und erst recht in der Verteidigung durch überzeugende Argumentation gewinnen. Sei es mit dem verbalen Zweihänder oder dem spitz formulierten Florett: Er war nie so stark, wie wenn er persönliche Angriffe scheinbar emotionslos an sich abprallen liess und darauf im charismatischen Gegenangriff eine ganze Ärztekammerversammlung hinter sich vereinigen konnte. Er wusste um diese Fähigkeit und verstand es, sie einzusetzen.

*

Was hat ihn in den letzten Jahren seiner Präsidentschaft bewegt? Je mehr er sich in die Analyse von Daten und Datenströmen vertiefte, desto mehr gingen seine Interessen über das eigentliche Tarifsystem hinaus. Dieses bildet ja nur einen Aspekt des Gesundheitswesens ab. Nein, er wollte, er suchte das Ganze. In gewissem Sinn war er ein später Nachfolger der Aufklärung, der versuchte, der Vernunft Raum zu schaffen, indem er dem unübersichtlichen Chaos des Gesund- 


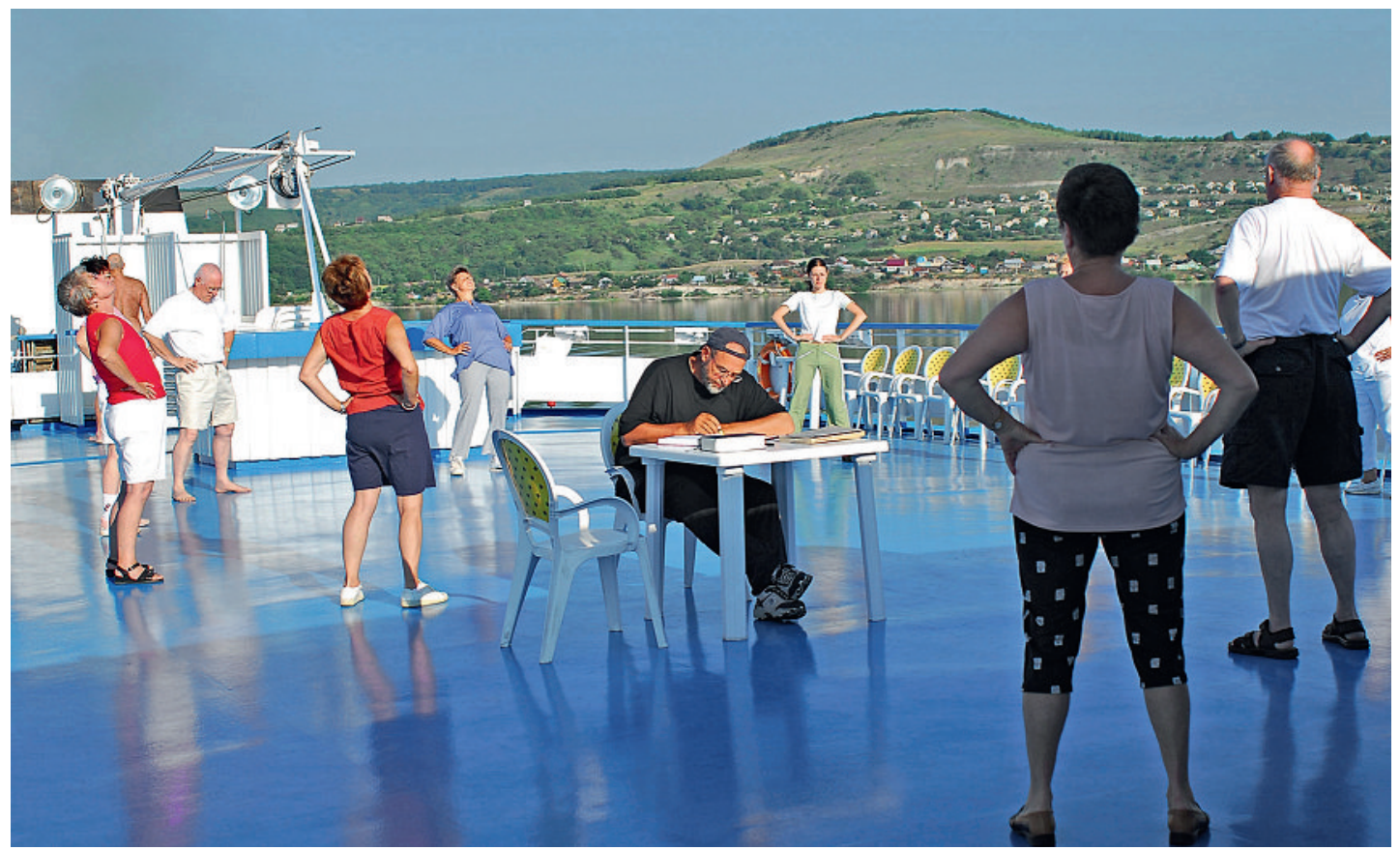

heitswesens eine gewiss nicht göttliche, aber zumindest innere Ordnung zu entlocken suchte. So vertiefte er sich in Studium und Entwicklung von medizinischen Leistungs-, Qualitäts- und Versorgungssystemen, untersuchte das «medical decision making» und absolvierte in den USA nebenher ein Masterstudium an der Harvard School of Public Health, um sich den theoretischen und auch akademischen Unterbau seiner gesundheitsökonomischen Visionen zu verschaffen. Das Generieren, Vergleichen und Kompilieren von Datenströmen schien gelegentlich zu einer «idée fixe» zu werden, die seine Umgebung zunehmend vor den Kopf stiess. Es wurde kühler um ihn, er traf seine Entscheidungen einsamer, erratischer, wie ein Bergsteiger, der sich in der Wand verstiegen hatte, drängte er nach vorn, ohne sich umzusehen. Der plötzliche, wie ein «Deus ex machina» erscheinende Wechsel vom Präsidentenstuhl der FMH in den Sessel des Vizedirektors im Bundesamt für Gesundheit im Jahr 2004 lässt sich rückblickend als konsequenter Schritt verstehen, als Versuch, die hundertköpfige Hydra des Gesundheitswesens von einer anderen Seite her in den Griff zu bekommen. Doch die persönlichen Angriffe und Beschuldigungen, die seinen Weggang von der FMH begleitet hatten, waren nicht ohne Folgen geblieben. Es war auch ein Verletzter, ein, wie er sich sah, Missverstandener und Gedemütigter, der sich ins Bundesamt zurückzog. Dass er dort noch weit weniger Handlungsfreiheiten und Gestaltungsmöglichkeiten hatte, musste er rasch erkennen. Schon 2006 quittierte er auch hier den Dienst, um sich wieder vermehrt der klinischen Tätigkeit zuzuwenden.

Hans Heinrich Brunner hatte viele Facetten. In einem ist er sich treu geblieben: Er war mit Leib und Seele Arzt. Sowohl als FMH-Präsident als auch als Vizedirektor des BAG behielt er ein Teilzeitpensum als
Oberarzt auf der Notfallstation des Inselspitals. Er wollte auch physisch mit dem Beruf in Berührung bleiben, weiterhin wissen und verstehen, worum es im medizinischen Alltag ging, wollte den Verhandlungspartnern nicht nur als Funktionär, sondern als Arzt gegenübersitzen. Es war, wie er es formulierte, seine «Freizeit», die er im angestammten Beruf verbrachte, meistens nachts oder an Feiertagen, immer auf Kosten der eigenen Erholungs- oder Ruhezeiten. Ein übermenschliches Pensum hat er sich oftmals zugemutet: tagsüber politische Verhandlungen, nachts Dienst auf der Notfallstation, dazwischen vier Stunden Schlaf, zur Entspannung die Lektüre von Heidegger und am Wochenende eine Bergtour. Im Umgang mit sich selbst konnte er masslos sein. Dass vieles dabei zu kurz kam, lag auf der Hand, aber seine immense Arbeitskraft nötigte Respekt ab. Er behielt die Anforderung an sich selbst bei, solange er konnte, zuletzt auch im Wechsel zwischen der Tätigkeit am tertiären Universitätszentrum und der neu gewonnen Aufgabe als Landarzt im Bergell. Niemand sollte ihn in die Knie zwingen, ausser zuletzt die krankheitsbedingte Schwäche des eigenen Körpers.

\section{*}

Am 11. Mai 2010 ist Hans Heinrich Brunner an den Folgen einer Berufskrankheit, die er sich als junger Arzt zugezogen hatte, verstorben. Die schweizerische Ärzteschaft verdankt ihm viel. Sofern es an einzelnen Stellen noch nötig ist, hat sie allen Grund, mit ihm Frieden zu schliessen und ihm ein ehrendes Andenken zu bewahren.

PD Dr. med. Ludwig T. Heuss, Chefarzt Medizinische Klinik Spital Zollikerberg, Mitglied des Zentralvorstands der FMH 1994-2006 